\title{
Sustainable Retrofit Design of Natural Gas Power Plants for Low Grade Energy Recovery
}

\author{
Qiao Q. Tang, Chang He, Qing L. Chen, Bing J. Zhang* \\ School of Materials Science and Engineering, Guangdong Engineering Center for Petrochemical Energy \\ Conservation, Sun Yat-Sen University, No.135, Xingang West Road, Guangzhou, 510275, China
}

*Corresponding author e-mail:

zhbingj@mail.sysu.edu.cn (Bing J. Zhang). 
Table S1. The specification of gas streams.

\begin{tabular}{cccc}
\hline Items & Mass Flowrate $(\mathrm{t} / \mathrm{h})$ & Temperature $\left({ }^{\circ} \mathrm{C}\right)$ & Pressure $(\mathrm{MPa})$ \\
\hline PNG & 5 & 0 & 4.0 \\
LNG & 20 & -100 & 2.5 \\
\hline
\end{tabular}

Table S2. The specification of cold energy entering users.

\begin{tabular}{ccccc}
\hline Items & Quantity $(\mathrm{MW})$ & Distance $(\mathrm{m})$ & Inlet temperature $\left({ }^{\circ} \mathrm{C}\right)$ & Outlet temperature $\left({ }^{\circ} \mathrm{C}\right)$ \\
\hline User\#1 & 12 & 1000 & 6 & 13 \\
User\#2 & 8 & 3000 & 8 & 12 \\
User\#3 & 10 & 5000 & 7 & 15 \\
\hline
\end{tabular}

